\title{
Frequency and trends of Hepatitis $B$ \& $C$, and human immune deficiency virus in blood donors presenting to blood bank of a Tertiary Hospital in Peshawar, Pakistan.
}

1. MBBS, M.Phil (Hematology) Assistant Professor Hematology Khyber Medical College.

2. MBBS, FCPS

Consultant Hematology Khyber Teaching Hospital.

3. MBBS, M.Phil (Chemical Pathology) Assistant Professor Chemical Pathology

Federal Medical \& Dental College.

Correspondence Address:

Dr. Muhammad Ihtesham Khan

Department of Hematology

Khyber Medical College.

ihteshamkhan9@yahoo.com.

Article received on:

$14 / 05 / 2020$

Accepted for publication:

06/07/2020

\begin{abstract}
Muhammad Ihtesham Khan', Neelam Ahmed ${ }^{2}$, Saman Waqar ${ }^{3}$
\end{abstract}
ABSTRACT... Objective: To determine frequency and trends of hepatitis $B$ \& $C$ and Human Immune deficiency Virus (HIV) in blood donors presenting to the blood bank of a tertiary hospital. Study Design: Cross Sectional Descriptive study. Setting: Blood Bank of Khyber Teaching Hospital, Peshawar. Period: January 2014 to October 2019. Material \& Methods: A total of 119263 blood bags were screened during the study period. Donors of age 18-55 years age and weight above 55 kilograms were included in the study. Cases who were drug addicts, anemic and jaundiced were excluded from the study. All blood bags were screened for Hepatitis B \& C and HIV using automated ELISA Cobas E-114 equipment. The data was analysed and results were calculated in terms of percentages. Results: A total of 119263 blood donors presented to the blood bank during the study period. Age range of the study sample was 20-45 years with mean of $31 \pm 7$ years. There were $119193(99.94 \%)$ males and $70(0.06 \%)$ females. The overall frequency of hepatitis B, hepatitis C and HIV was $1.42 \%, 0.76 \%$ and $0.018 \%$ respectively. The frequency of these infections was lowest in 2015 and highest in 2016. The trend of hepatitis B increased while that of hepatitis C decreased from 2014 to 2019. Conclusion: Hepatitis B infection is more prevalent in blood donors as compared to hepatitis $C$ and HIV in our region. Actions should be taken to create awareness in population about the spread of hepatitis $B$ and strict screening of the blood donors should be ensured to stop the rise in incidence of hepatitis $B$ in our population.

Key words: $\quad$ Blood Bank, Blood Donors, Hepatitis B, Hepatitis C, HIV.

Article Citation: Khan MI, Ahmed N, Waqar S. Frequency and trends of Hepatitis B \& C, and human immune deficiency virus in blood donors presenting to blood bank of a Tertiary Hospital in Peshawar, Pakistan. Professional Med J 2021; 28(4):515-519. https://doi.org/10.29309/TPMJ/2021.28.04.4798

\section{INTRODUCTION}

Blood transfusion services save millions of lives on day to day basis all over the world by providing blood and blood products to the needy. ${ }^{1-3}$ No doubt blood transfusion is life saving procedure, but if the blood is unscreened or infected, it can transmit a number of fatal infections within general population. ${ }^{3-5}$ Hepatitis $\mathrm{B}, \mathrm{C}$ and HIV are the most common infections that can be transmitted by transfusion of unsafe or infected blood and blood products. $^{3-5}$ The transmission of these blood borne infections is a big health problem all over the world especially in the developing countries like Pakistan .These infections are transmitted from person to person in community and increases the morbidity and mortality of the population. ${ }^{6,7}$

Worldwide, about 250 million people are infected with hepatitis B virus and about 150-200 million are infected with hepatitis $C$ virus. ${ }^{1,8,9}$ The World Health Organization reported that in 2015, there were about 71 million people living with chronic hepatitis $\mathrm{C}$ and about 36 million people suffering from HIV all over the world. ${ }^{5}$ The prevalence of these infections is increasing not only in the Western countries but in Pakistan as well. ${ }^{1}$ The estimated worldwide prevalence of hepatitis $B$, $\mathrm{C}$ and HIV among blood donors according to the World Health Organization is $6.08 \%, 1.96 \%$, and $2.0 \%$, respectively. ${ }^{6}$

Pakistan is a developing country where the morbidity and mortality due to hepatitis B and C is on the rise. ${ }^{10}$ It is reported that about 1 million transfusions are done in Pakistan each year. ${ }^{11}$ It is reported that in Pakistani population, the 
prevelance of hepatitis $\mathrm{B}$ and $\mathrm{C}$ is about $2.5 \%$ and $4.8 \%$ respectively. ${ }^{9}$ Literature suggests that the incidence of hepatitis $\mathrm{C}$ is gradually increasing in Pakistan. ${ }^{10}$ About 10 million people are reported to be hepatitis $C$ positive in our country. ${ }^{10}$ However, the prevalence of HIV in Pakistan is comparatively low i.e $0.1 \% .^{1,11}$ Although the prevelance of hepatitis $B$ and $C$ is high in Pakistani population, yet most of them are not aware that they are infected. ${ }^{1}$ Most of the cases with theses infections come to know about their disease status accidentally when they donate blood and it is screened in the blood bank. ${ }^{1}$

It is reported that all over the world, about 80 million blood bags are donated annually. ${ }^{1}$ Out of these, about 18 million of the blood bags are not screened for transfusion related infections. ${ }^{1}$ With every blood bag being transfused, there is about $1 \%$ chance that it will transmit an infection to its recipient. ${ }^{1}$ The $\mathrm{WHO}$ has rendered it mandatory to screen the donated blood bags for blood borne infections like Hepatitis B, hepatitis C, HIV, syphilis and malaria. ${ }^{13}$

The present study was done in order to determine the prevalence of hepatitis B\& C, and HIV , and to study the trends of these infections in our region. This will help us to assess the disease burden and its trends in our region.

\section{MATERIAL \& METHODS}

This cross sectional descriptive study was carried out in blood bank of Khyber Teaching Hospital Peshawar from January 2014 to October 2019. The blood donors aged 18 -55 years of both the genders were included in the study. Donors who were drug addicts, alcoholics, underweight anemic and those who had donated blood in the preceding three months were not included in the study. At the blood bank, a well trained phlebotomist was assigned to take $2 \mathrm{~mL}$ blood sample from the antecubital vein of the donors in EDTA tube. The serum was separated from it by centrifugation in a centrifuge machine at $15000 \mathrm{rpm}$. The serum was screened for HBs antigen (for hepatitis B), anti HCV antibodies (for Hepatitis C) and anti HIV antibodies (for HIV) through automated ELISA Cobas E-114 equipment which works on chemoilluminicance principle. It was ensured that controls are run with each batch so that the sensitivity may not be compromised. The data thus piled through all the years from 2014 to 2019 was first completely anonymized before analyzing it. This was done to ensure that the privacy of every participant is kept secret. The hospital ethical committee was informed about the data being anonymized and that there is no risk for the breach of privacy of participants. Keeping in mind the anonymity of the data, the ethical committee granted the approval for the study. The data was analyzed. The results for hepatitis $B, C$ and HIV were noted and their rates were analysed using percentages .Quantitative variables were measured by using mean and standard deviation. Qualitative data was analysed using frequency and percentages. The prevalence of each year was compared among different groups for significance by using chi square test. A p value of less than 0.05 was taken as statistically significant. The prevalence trends were plotted using scatter diagram.

\section{RESULTS}

A total of 119263 blood donors presented to the blood bank during the study period.

Age range of the study sample was 20-47 years with mean of $31 \pm 7$ years.

There were 119193 (99.94\%) males and 70 $(0.06 \%)$ females.

The overall frequency of hepatitis $B$, hepatitis $C$ and HIV is shown in Table-I.

The trends of these infections are shown in Figure-1.

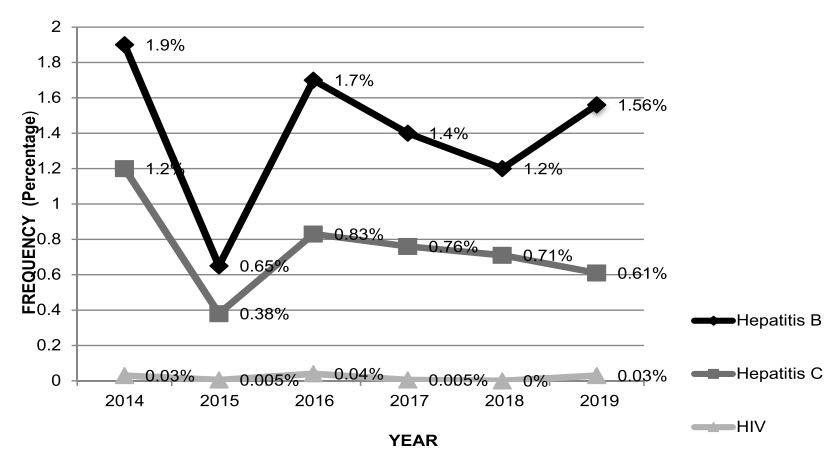

Figure-1 


\begin{tabular}{|l|c|c|c|c|}
\hline \multicolumn{1}{|c|}{ Year } & Total Blood Screened & Hepatitis B Positive & Hepatitis C Positive & HIV Positive \\
\hline 2014 & 18812 & $361(1.9 \%)$ & $235(1.2 \%)$ & $1(0.03 \%)$ \\
\hline 2015 & 19314 & $126(0.65 \%)^{\star}$ & $74(0.38 \%)^{\star}$ & $9(0.04 \%)^{\star}$ \\
\hline 2016 & 22695 & $394(1.7 \%)$ & $188(0.83 \%)$ & $1(0.005 \%)^{\star}$ \\
\hline 2017 & 19760 & $283(1.4 \%)$ & $152(0.76 \%)$ & Nil \\
\hline 2018 & 22792 & $283(1.2 \%)$ & $162(0.71 \%)$ & $5(0.03 \%)^{\star}$ \\
\hline 2019 & 15890 & $248(1.56 \%)$ & $908(0.76 \%)$ & $22(0.018 \%)$ \\
\hline Grand total & 119263 & $1695(1.42 \%)$ & .025 & .033 \\
\hline P value & & .000 &
\end{tabular}

\section{DISCUSSION}

The blood transfusion is a life saving procedure. However, if the blood being transfused is not screened for blood borne infections and is unsafe, it can be a source of spread of infections from donors to general population. ${ }^{1}$ Hepatitis $\mathrm{B}, \mathrm{C}$ and HIV are common infections that are frequently transmitted because of transfusing unsafe or unscreened blood to the population. ${ }^{3}$ If the screening of the blood bags is ensured before transfusing them to the population, the transmission of these infections can be stopped. ${ }^{1}$ This will also decrease the burden of these infections in community. ${ }^{1}$

In our research, the overall prevalence of hepatitis $\mathrm{B}, \mathrm{C}$ and HIV was $1.56 \%, 0.76 \%$ and $0.018 \%$ respectively. The prevalence of hepatitis $B$ was highest and that of HIV was lowest comparatively. The trend of these infection was on increase from 2014 to 2015 and then it decreased upto 2019 except hepatitis B which showed a spike in 2019. A study done by Zahoor M from Peshawar showed that prevalence of hepatitis B,C and HIV in blood donors are $1.6 \%, 1.1 \%$ and $0.16 \%$ respectively, showing that prevalence of hepatitis B was highest. ${ }^{1}$ Similar data is reported by Ahmad from Peshawar in 2017. ${ }^{14}$ However, a study done in Kyrgyzistan showed the prevelance of hepatitis B,C and HIV was $3.6 \%, 3.1 \%$ and $0.78 \%$ respectively. ${ }^{15}$ These rates are quite higher as compared to that reported in our study. Similarly, predominance of hepatitis $B$ in blood donors was reported by a study done in Karachi by Arshad and from Peshawar by Ahmad T. ${ }^{16,17}$ Predominance of hepatitis B was reported from Africa as well. ${ }^{18}$
However, Jiskani SA from Sindh, Ali ST from Hyderabad, Pervaiz A, Humayun, and Mujeed reported hepatitis $C$ to be more common than hepatitis $\mathrm{B}$ in blood donors in their studies. ${ }^{3,19-22}$ Zaheer also reported hepatitis $C$ to be more common in donors as compared to hepatitis B. ${ }^{11}$ In a study done by Shifarew in 2019 from Ethopia, prevalence of hepatitis B was $6 \%$ which is quite high figure. ${ }^{5}$ In the same study, it was reported that the prevelance of hepatitis $B$ increased while that of hepatitis C and HIV decreased from 2014 to $2018 .{ }^{5}$ This trend is similar to that reported in our study. The overall prevalence of blood born infections in blood donors in the present study was $2.3 \%$. This is quite low as compared to other studies where the figures of $6 \%, 5.29 \%, 6.55 \%$ and $7.1 \%$ are reported. ${ }^{5,23-25}$

The WHO stresses upon that all the blood bags should be screened for hepatitis B, C, HIV and syphilis. ${ }^{3}$ There are different methods that are used currently for screening of the blood i.e immunochromatography method (ICT), ELISA method and Nucleic acid testing method (NAT). ${ }^{26}$ The ICT method is less sensitive while the NAT method is the most advanced and most sensitive method so far. Screening of the blood should be done by any of these methods. However, the sensitivity of diagnosis increases if ELISA or NAT is used.

\section{CONCLUSION}

The prevalence of hepatitis $B$ is more as compared to hepatitis $C$ and HIV in our region. This should alert the health care providers. Blood bags should be screened before transfusing them 
to the recipients to control the spread of these infections to general population.

\section{RECOMMENDATION}

This study shows that there is a need to create awareness in general population about hepatitis $B$ and its spread. Donated blood should be screened before transfusiong them to the population. Also, studies should be conducted in hospitals time to time to keep an eye on prevalence and trends of these blood born infections in future. This will help to monitor the effectiveness of our screening systems and donor selection.

Copyright@ 06 July, 2020.

\section{REFERENCES}

1. Zahoor M, Zahoor S, Khan K, et al. Seroprevalence of Hepatitis B,C and HIV infection in healthy blood donors; A single centre study in Peshawar. KJMS 2019; 12(2):192-5.

2. Shah N, Shah JM, Jhaveri P, et al. Seroprevalence of HBV, HCV, HIV and syphilis among blood donors at a tertiary Care Teaching Hospital in Western India. G Med J 2013; 68(2):35-9.

3. Jiskani SA, Talpur RA, Meghji KA, et al. Seropositivity of hepatitis $\mathbf{B}$ and $\mathbf{C}$ among volunteer blood donors at Tando Muhammad Khan. Pak J Pathol 2019; 30(3):6971.

4. Alzoubi $\mathrm{KH}$. The attitude of blood donors towards the use of their samples and information in biomedical. J Blood Med 2018; 9:145-51.

5. Shiferaw E, Tadilo W, Melkie I, et al. Sero-prevalence and trends of transfusion-transmissible infections among blood donors at Bahir Dar district blood bank, northwest Ethiopia: A four year retrospective study. PLoS ONE 2019:14(4):e0214755.

6. Farshadpour F, Taherkhani R, Tajbakhsh S, et al. Prevalence and trends of transfusion- transmissible viral infections among blood donors in South of Iran: An eleven-year retrospective study. PLoS One 2016; 16;11(6):e0157615. doi:10.1371/journal.pone.0157615. eCollection2016.

7. Abdela A, Woldu B, Haile K,et al. Assessment of knowledge, attitudes and practices toward prevention of hepatitis $B$ virus infection among students of medicine and health sciences in Northwest Ethiopia. BMC Res Notes 2016; 9(1):410.
8. Ashraf S, Ahmad A. Viral hepatitis in Pakistan: Challenges and priorities. Asian Pac J Trop Biomed 2015; 5(3):190-1.

9. Hussein NR, Haj SM, Almizori LA, et al. The prevalence of Hepatitis $B$ and $C$ viruses among blood donors attending blood bank in Duhok, Kurdistan Region, Iraq. Int J Infect 2017; 4(1):1-6.

10. Khan MI, Rahman SU, Salman. Knowledge and practices about viral hepatitis in blood bank staff at a tertiary care transfusion centre in Islamabad. J Med Sci 2017; 25:(4):409-13.

11. Zaheer HA, Waheed U. Blood safety system reforms in Pakistan. Blood Transfus 2014; 12:452-7.

12. Ismail S, Awan S, Naeem R, et al. Occupational exposure to HIV in a developing country: Assessing knowledge and attitude of healthcare professional before and after an awareness symposium. BMC Res Notes 2018; 11:131.

13. Zameer M, Shahzad F, Khan SF, et al. Transfusion transmissible infections among healthy blood donors at blood bank from children's hospital \& institute of child health Lahore. Pak Armed Forces Med J 2017; 67(1):131-6.

14. Ahmad T, Nadeem M, Khan FU, et al. Incidence of HV, HCV and HIV among blood donors from Peshawar KPK, Pakistan. J Entomol zool stud 2017; 5(4):608-10.

15. Karabaev BB, Beisheeva NJ, Satybaldieva AB, et al. Seroprevalence of hepatitis $B$, hepatitis $C$, human immunodeficiency virus, Treponemapallidum, and coinfections among blood donors in Kyrgyzstan: A retrospective analysis. Infect Dis Poverty 2017; 6:45.

16. Arshad A, Borhany M, Anwar N, et al. Prevalence of transfusion transmissible infections in blood donors of Pakistan. BMC Hematology 2016; 16:27.

17. Ahmed T, Khattak NN, Khan F, et al. Prevalence of Hepatitis B virus, Hepatitis C virus and HIV in blood donors of different areas of Khyber Pukhtoonkhwa, Pakistan. J Bio Env Sci 2016; 9(1):304-9.

18. Mohammed $Y$, Bekele A. Seroprevalence of transfusion-transmitted infection among blood donors at Jijiga blood bank, Eastern Ethiopia: Retrospective 4 years study. BMC Res Notes 2016; 9:129.

19. Ali TS, Shaista B, Ali LZ, et al. Seroprevalence of Hepatitis $B$ and $C$ among blood donors in Hyderabad, Pakistan. Gomal Med J Sci 2013; 11(2):220-3. 
20. Pervaiz A, Sipra FS, Rana TH, et al. Pre-Donation screening of volunteer prisoner blood donors for Hepatitis B and C in Prisons of Punjab, Pakistan. J Ayub Med Coll Abottabad 2015; 27(4):794-7.

21. Ayesha $H$, Ameena A, Afsar $S$, et al. Knowledge of hepatitis $B$ and $C$ infections and sero-prevalence among blood donor university students in District Lahore. Annals 2011; 17(4):371-8.

22. Mujeeb SA, Pearce MS. Temporal trends in hepatitis $B$ and $\mathbf{C}$ infection in family blood donors from interior Sindh, Pakistan. BMC Infect Dis 2008; 6(43):1-6.

23. Sadiq MA, Hashim R, Hussain A, et al. Frequency of hepatitis $B$ and $C$ virus among healthy blood donors .A single centre study. Pak J Pathol 2017; 28(3):105-8.
24. Biadgo B, Shiferaw E, Woldu B, et al. Transfusiontransmissible viral infections among blood donors at the North Gondar district blood bank, Northwest Ethiopia: A three year retrospective study. PloS one 2017; 12(7):e0180416.

25. Misganaw B. Prevalence of transfusion-transmissible infections in donors to an Ethiopian blood bank between 2009 and 2013 and donation factors that would improve the safety of the blood supply in underdeveloped Countries. Lab med 2016; 47(2):1349.

26. Farooq $A$, Waheed $U$, Zaheer HA, et al. Detection of HBsAg mutants in the blood donor population of Pakistan. PLoS One 2017; 12(11):1-11.

\begin{tabular}{|c|c|c|c|}
\hline \multicolumn{3}{|c}{ AUTHORSHIP AND CONTRIBUTION DECLARATION } \\
\hline Sr. \# & Author(s) Full Name & \multicolumn{1}{|c|}{ Contribution to the paper } & Author(s) Signature \\
\hline 1 & M. Ihtesham Khan & $\begin{array}{l}\text { Main idea, Data collection, } \\
\text { Principle author. } \\
\text { Data compilation, Data } \\
\text { analysis. } \\
\text { Discussion, Review, Literature } \\
\text { review. }\end{array}$ \\
\hline 3 & Saman Waqar & Neelam Ahmed & S \\
\hline
\end{tabular}

